ARTICLE

\title{
The cichlid oral and pharyngeal jaws are evolutionarily and genetically coupled
}

\author{
Andrew J. Conith (10) ${ }^{1 凶} \&$ R. Craig Albertson (10 ${ }^{1 凶}$
}

Evolutionary constraints may significantly bias phenotypic change, while "breaking" from such constraints can lead to expanded ecological opportunity. Ray-finned fishes have broken functional constraints by developing two jaws (oral-pharyngeal), decoupling prey capture (oral jaw) from processing (pharyngeal jaw). It is hypothesized that the oral and pharyngeal jaws represent independent evolutionary modules and this facilitated diversification in feeding architectures. Here we test this hypothesis in African cichlids. Contrary to our expectation, we find integration between jaws at multiple evolutionary levels. Next, we document integration at the genetic level, and identify a candidate gene, smad7, within a pleiotropic locus for oral and pharyngeal jaw shape that exhibits correlated expression between the two tissues. Collectively, our data show that African cichlid evolutionary success has occurred within the context of a coupled jaw system, an attribute that may be driving adaptive evolution in this iconic group by facilitating rapid shifts between foraging habitats, providing an advantage in a stochastic environment such as the East African Rift-Valley.

${ }^{1}$ Biology Department, University of Massachusetts Amherst, Amherst, MA 01003, USA. 凶email: ajconith@bio.umass.edu; albertson@bio.umass.edu 
$\mathrm{T}$ he potential phenotypes available to a population are not limitless, rather, their bounds are shaped by various genetic, developmental, environmental, and functional constraints $^{1,2}$. Constraints that act upon the phenotype can impact certain evolutionary metrics such as phenotypic disparity, and rates of taxonomic and morphological evolution, that may ultimately determine the "success" of a given clade ${ }^{3-5}$. Breaking from constraints involves natural selection navigating a very specific mutational landscape, but in doing so, may improve functional efficiency, or enable a population to access unoccupied ecological niche space, ultimately fostering subsequent diversification ${ }^{6}$. Therefore, constraints, when broken, can lead to rapid evolutionary change in a clade and may be responsible for the unevenness in rates of taxonomic and morphological evolution observed across metazoans, providing an opportunity to explore incipient stages of diversification ${ }^{7,8}$.

Evolutionary constraints are ubiquitous across organisms and can influence the degree of variation in a trait, or the strength of covariation among traits ${ }^{9,10}$. Weak evolutionary constraints acting among traits could lead to many trait possibilities as each trait varies independently becoming more modular, while strong constraints may lead to fewer possibilities as traits become integrated (Fig. 1a). But how a population moves in a morphospace, with ranges defined by the strength of various constraints, is ultimately determined by the direction and magnitude of selection. Indeed, selection for shared functions or genetic pleiotropy can produce strong integration among traits, biasing phenotypic evolution to occur along a narrow line delimited by the covariation among traits--the line of least resistance ${ }^{11,12}$. When an evolutionary constraint is broken, some populations may deviate from this line as selection acts upon each trait independently (Fig. 1b), allowing each trait to become more specialized for a given task and permitting more unique trait combinations.

An iconic example of this process is illustrated by the feeding system in ray-finned fishes, whereby a second set of "pharyngeal" jaws have evolved by lining the posterior ceratobranchial bones of the gill arch with teeth, permitting the separation of prey capture and prey processing mechanisms (Fig. 1c-d). Functionally decoupling the jaw systems releases ray-finned fishes from mechanical constraints typically imposed by force-velocity tradeoffs in single jaw systems ${ }^{13}$; a single jaw cannot be optimized for both power and speed simultaneously, but a two jaw complex could optimize each jaw independently for one of power or speed $^{14}$. Within the ray-finned fishes, some clades of percomorph fishes (i.e., Cichlidae, Labridae, Pomacentridae, and Embiotocidae), have independently evolved further specializations to their pharyngeal jaws that increased feeding efficiency, specializations that are theorized to be key to their success ${ }^{15}$.

Cichlids are characterized by rapid speciation within a diverse array of ecological niches throughout the subtropics (SouthCentral America, Africa, India), and the lakes of the East African Rift Valley have fostered multiple adaptive radiations, particularly within lakes Malawi, Tanganyika, and Victoria ${ }^{16-18}$. Cichlids owe much of their success to a highly evolvable feeding system, and in particular, the specialized pharyngeal jaw apparatus that is thought to represent a "key innovation." Greater pharyngeal jaw efficiency arises from the evolution of two synovial joints between the upper pharyngeal jaw and basicranium, fused fifth ceratobranchials, and a direct muscular connection between the neurocranium and the lower pharyngeal jaw, which together permit a stronger bite force and is predicted to provide an advantage over interspecific competitors ${ }^{19}$. Liem initially formulated the functional decoupling hypothesis after seminal work describing the cranial anatomy and performing kinematics of cichlid feeding ${ }^{20,21}$. Following Liem's work, future studies hypothesized that, alongside functional modularity, the cichlid oral and pharyngeal jaws reflect separate genetic and developmental modules, permitting variation to accumulate in each pair of jaws independently and promoting trophic diversification ${ }^{22,23}$.

Documenting the degree of integration between oral and pharyngeal jaws across genetic, molecular, and developmental levels is crucial to formulating a more comprehensive

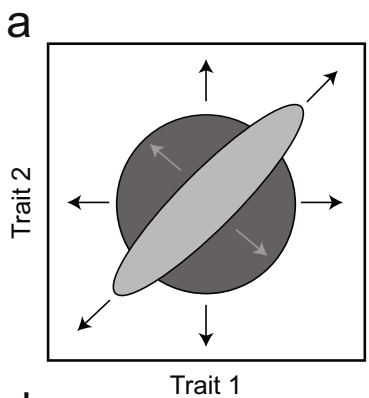

d

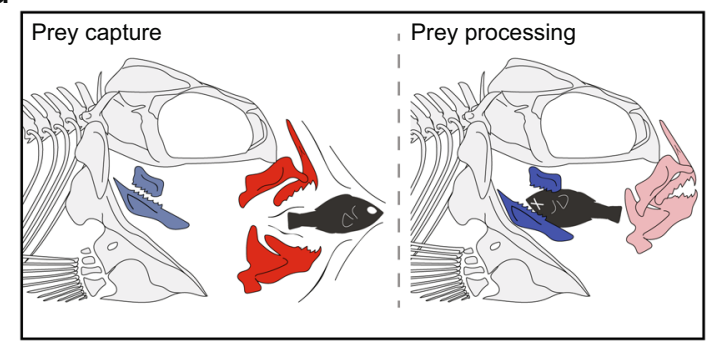

b

Trait 1

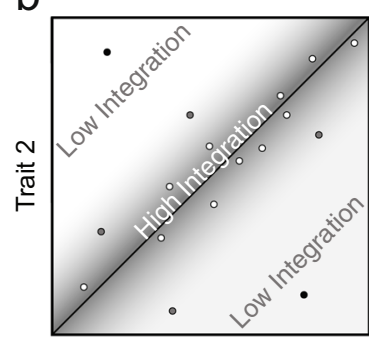

e

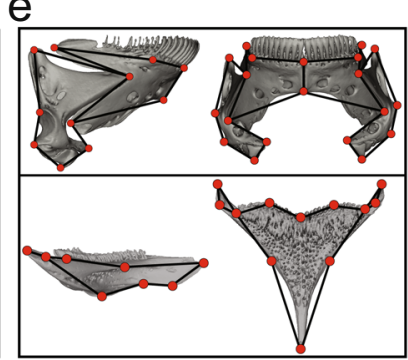

Fig. 1 The impacts of constraints on phenotypic evolution. a Dark gray, no constraints permitting phenotypic evolution to occur in any direction. Light gray, some constraints operating on trait evolution permitting selection to dive phenotypic change in some directions, but limiting change in others. b Assessing the degree of integration between traits would reveal the strength of covariation among taxa/individuals. c Schematic illustrating the relative positions of the oral (red) and pharyngeal (blue) jaws within the skulls of cichlid fishes. $\mathbf{d}$ Schematic illustrating the relative roles of each jaw complex in prey capture and prey processing. e Oral and pharyngeal jaw symmetric landmark configurations. Top, lower oral jaw $\mu \mathrm{CT}$ scans (lateral, anterior view). Bottom, lower pharyngeal jaw $\mu \mathrm{CT}$ scans (lateral, dorsal view). Full landmark configuration information can be found in the supplement. Scan images and line drawings were produced by the authors using MeshLab (v2019-12) and Adobe Illustrator CC (v22.0.1). 
understanding of what constraints have shaped cichlid evolutionary dynamics and permitted such explosive adaptive radiations. While there is some evidence to suggest the jaw systems are evolutionarily decoupled based on certain functional metrics ${ }^{22,24}$, other work has demonstrated a degree of evolutionary and genetic integration between the oral and pharyngeal jaws ${ }^{14,25-27}$. There is also a need in the field to assess integration at multiple taxonomic levels, as patterns at higher, macroevolutionary levels (i.e., between genera) may or may not be related to those at lower, microevolutionary levels (i.e., within species) or developmental levels (i.e., within individuals), owing to distinct genetic and evolutionary phenomena influencing the manifestation of patterns at each level. For example, examining associations across cichlids may reveal unique combinations of oral jaw and pharyngeal jaw traits in a minority of taxa that, while their jaws appear more decoupled (i.e., residing far from a line of best-fit), may still be integrated but represent a reversal of the direction of the correlation (Fig. 1a; association follows the perpendicular trajectory, light gray arrows of light gray ellipse). Examining jaw associations within cichlid taxa can complement such higher-level analyses by comparing (1) the strength of integration between species (Fig. 1b; narrow distribution versus wide distribution around best-fit line), and (2) the aspects of the anatomy that are co-varying in each lineage. For the purposes of this project, when we describe the relationship between the LOJ and LPJ of cichlids as 'integrated', we are referring to the strength of correlation between jaw shapes; however, we also provide descriptions of anatomical (co)variation so that patterns of integration may be assessed and compared between evolutionary levels.

There is evidence to suggest that cichlids can experience some variation in the degree of integration between their oral and pharyngeal jaws, and that integration itself may be dependent on feeding ecology ${ }^{14}$. Specifically, despite the perceived ability of cichlids to free themselves from mechanical constraints, cichlids that hunt elusive prey typically pair slender, mobile oral jaws with gracile pharyngeal jaws, while cichlids that feed on algae or other tough foods typically pair robust, compact oral jaws with strong pharyngeal jaws. This form-function relationship suggests feeding ecology may be the predominant force that determines integration between jaws. An alternative explanation is that the oral and pharyngeal jaws of cichlids are integrated as a consequence of some intrinsic genetic or developmental constraints such as pleiotropy $^{28}$, and that this precludes many cichlid taxa from exploring all the available functional combinations of jaw morphologies. Asserting this scenario posits that a handful of taxa, typically with specialist diets, have broken free of these constraints or exhibit unique oral-pharyngeal jaw association trajectories (e.g., lepidovores, molluscivores, ovivores etc.), and suggests decoupled oral and pharyngeal jaws are an exception, and not the rule.

Here we characterize the integration between cichlid lower oral jaw (LOJ) and lower pharyngeal jaw (LPJ) shapes at the genetic, phenotypic, and evolutionary level. We test the hypothesis that the oral and pharyngeal jaws of cichlids represent distinct phenotypic and genetic modules that would permit the exploration of independent evolutionary trajectories directed by selection. We employ an array of phylogenetic comparative methods, statistical genetics, and molecular approaches to demonstrate how certain aspects of shape variation in the oral and pharyngeal jaws of cichlid fishes are evolutionarily and genetically coupled. Our data roundly support the idea that the oral and pharyngeal jaws are integrated. Furthermore, we uncover a set of linked candidate genes that may contribute to this integration. By taking a multifaceted approach that assesses several levels of organismal diversity, from genes through morphology, function and resource-use, we gain a more holistic understanding of how adaptive variation is generated under constraint, and how constraints, or the lack-thereof, can contribute to our understanding of diversification 29,30 .

\section{Results}

Macro- and micro-evolutionary integration between jaw complexes. We examined phenotypic associations between the lower oral and pharyngeal jaws (LOJ and LPJ, respectively) of 88 cichlid species from across Africa, primarily sampling from lakes in the East African Rift Valley: lakes Malawi, Tanganyika, and Victoria (Supplementary Data 1). We characterized jaw shapes based on 107 individuals using 3D geometric morphometrics by placing landmarks in positions that capture functionally (e.g., bony processes, sutures, etc.) and developmentally (e.g., distinct cellular origins) important aspects of morphology, including placing mirrored landmarks across midlines to gain symmetric configurations (Fig. 1e, Supplementary Fig. 1). We conducted a Procrustes superimposition, removed the effects of allometry to account for size differences, and then removed the effects of asymmetry to account for developmental noise. We performed a two-block partial least squares (PLS) analysis on the species mean landmark configurations and corrected for phylogenetic nonindependence using a Bayesian time-calibrated tree ${ }^{31}$. We found the LOJ and LPJ were evolutionarily correlated ( $r-P L S=0.482$, $P=0.002$, effect size $(Z)=2.585$ ), but some taxa, particularly those with unique diets and/or modes of feeding, appeared to deviate from the best-fit line, indicating lower levels (or different patterns) of integration between jaws (Fig. 2a). Indeed, we found numerous taxa, typically from Lake Malawi, whereby covariation between the LOJ and LPJ appeared much different relative to other African cichlids. Taxa placed far from the best-fit line either (1) exhibited a specialized feeding morphology to better exploit an foraging niche shared with many competitors (i.e., Labeotropheus, algae; Copadichromis, zooplankton; Taeniolethrinops, insects), or (2) exhibited a specialized feeding morphology to take advantage of a more challenging food source (i.e., Trematocranus, snails). However, not all taxa that consume specialized prey were far from the best-fit line; Pungu, (primarily a sponge-feeder) and Perissodus (a scale-feeder), while exhibiting specialized feeding apparatuses to consume such prey, exhibited a relationship between their LOJ and LPJ that was in-line with other African cichlids (Supplementary Fig. 2). We also noted, that while Malawi cichlids exhibit a range of LOJ-LPJ relationships (from weak to strong), most Tanganyikan cichlids reside close to the best-fit line. However, when we examine the strength of integration in the Tanganyika group $(n=29, \mathrm{r}-\mathrm{PLS}=0.698, P=0.001, Z=2.954)$ and Malawi group ( $n=40, \mathrm{r}$-PLS $=0.541, P=0.020, Z=2.155)$, despite Tangyanika cichlids exhibiting higher $Z$-scores, consistent with stronger integration, a statistical comparison between groups finds no significant difference ( $\mathrm{Z}$ pairwise $=1.188, P=0.235$ ). Comparisons between Tanganyikan and Malawi cichlids should not be influenced by sampling bias, as principal components analyses (PCA) on the LOJ and LPJ landmark data (Supplementary Data 2 and 3) showed that our sampling of Tanganyikan cichlids includes many species with extreme morphologies that reside at the outer edges of LOJ and LPJ morphospace (Supplementary Fig. 3). Indeed, cichlids from Lake Tanganyika exhibited similar LOJ morphological disparity (Malawi Procrustes variance $(P V)=0.074$; Tanganyika $P V=0.057, P=0.253)$ and greater LPJ morphological disparity (Malawi $\mathrm{PV}=0.015$; Tanganyika $\mathrm{PV}=$ $0.023, P=0.012$ ), relative to cichlids from Lake Malawi. Taken together, this indicates that while Tanganyikan cichlids exhibit comparable (i.e., LOJ), or greater (i.e., LPJ) morphological variation compared to Malawi cichlids, covariation between LOJ and LPJ shapes was generally similar between groups. 

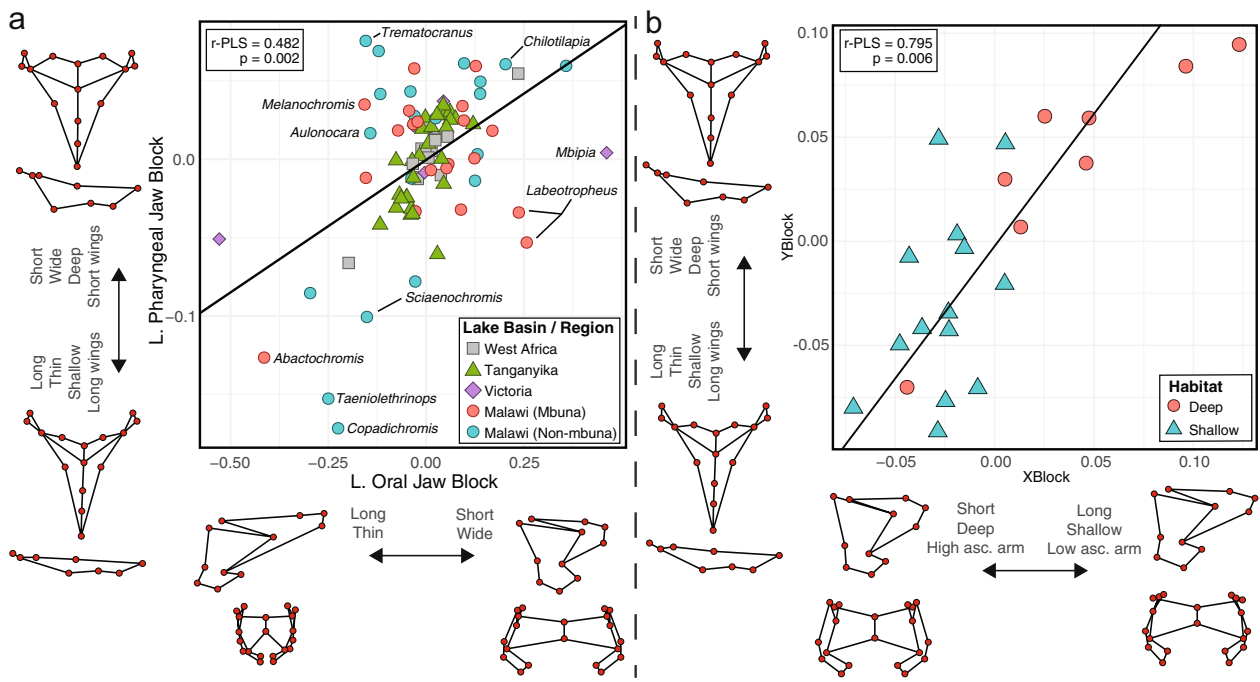

Fig. 2 Phylogenetic two-block partial least squares analysis to assess macroevolutionary associations between lower oral and pharyngeal jaws. a Jaw shape associations across a broad sample of African cichlids $(n=88)$. Taxa from Lake Malawi are placed into two groups based on phylogenetic position: an mbuna 'rock-dwellers' group, and a non-mbuna group consisting of the utaka 'sand-dwellers' alongside other benthic species ${ }^{88}$. b Jaw shape associations across the Tropheops sp. species complex from across a depth gradient $(n=22)$. Oral and pharyngeal jaw wireframes denote morphologies at either end of the correlational axis. Source data are provided as a Source Data file.
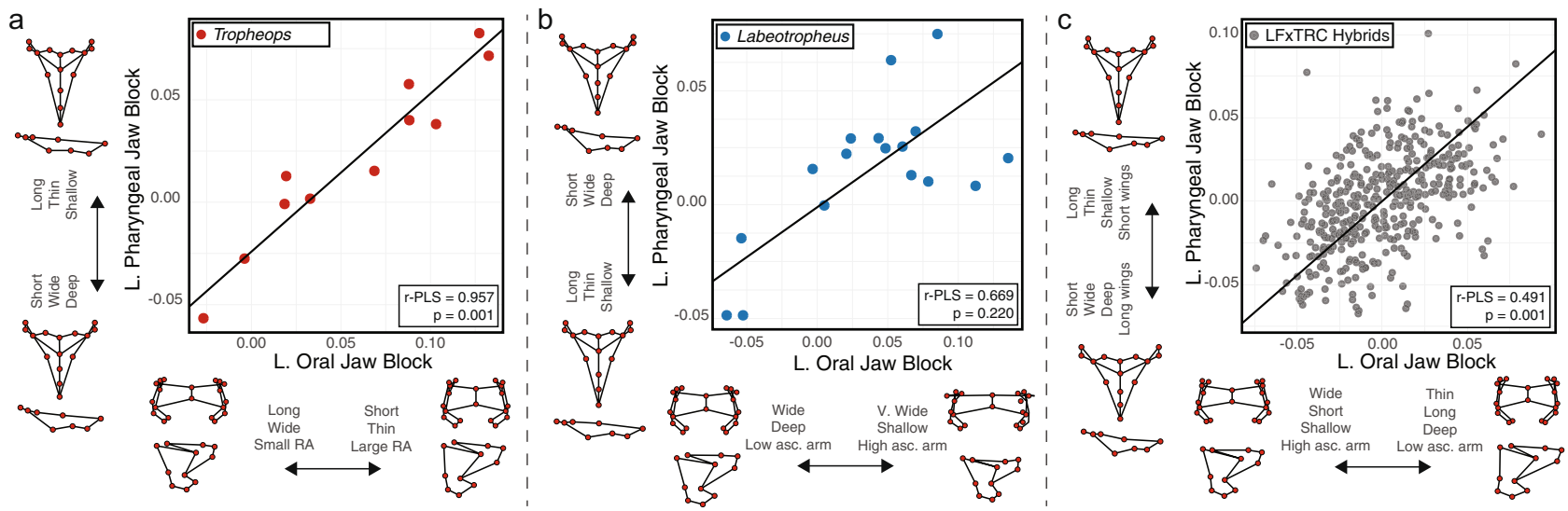

Fig. 3 Two-block partial least squares analysis to assess microevolutionary associations between lower oral and pharyngeal jaws. a Shape associations among Tropheops sp. "red cheek" (TRC) individuals $(n=11)$. b Shape associations among Labeotropheus fuelleborni (LF) individuals $(n=17)$. c Shape associations among members of a hybrid cross between TRC and LF $(n=409)$. Oral and pharyngeal jaw wireframes denote morphologies at either end of the correlational axis. Source data are provided as a Source Data file.

We next investigated the degree of integration at lower taxonomic levels. First, we analyzed the jaws of cichlids within the Tropheops species complex from Lake Malawi that is diverse and known to partition habitat by depth ${ }^{32,33}$. While Tropheops exhibited strong integration between jaws in on our macroevolutionary assessment, species within this genus occupy a broader niche space. Investigating integration within such a species complex provided an opportunity to understand whether habitat differences could lead to differences in integration between jaw complexes. Using the same landmarking procedure as described above, we characterized shape variation in the LOJs and LPJs of 22 wild-caught Tropheops taxa from 60 individuals, concentrating on members from localities across the southern portion of Lake Malawi (Supplementary Data 4). We again performed a twoblock PLS analysis on the mean landmark configurations and accounted for phylogenetic non-independence using an amplified fragment length polymorphism tree ${ }^{33}$. Again, we found the LOJ and LPJ were evolutionarily correlated ( $\mathrm{r}-\mathrm{PLS}=0.795, P=0.006$,
$Z=2.521$ ), indicating jaw integration does not appear to vary by habitat (Fig. 2b).

Finally, we measured and compared integration between a species pair that exhibited relatively strong versus weak covariation between LOJ and LPJ shapes in our macroevolutionary assessments, Tropheops sp. 'red cheek' (TRC, relatively stronger integration) and Labeotropheus fuelleborni (LF, relatively weaker integration). Using the same landmarking protocol we performed separate two-block PLS analyses between LOJs and LPJs of LF and TRC (Supplementary Data 5). Notably, we found strong and significant integration between jaw complexes in TRC $(n=11, \mathrm{r}$ $\mathrm{PLS}=0.957, P=0.001, Z=3.038$; Fig. $3 \mathrm{a})$ relative to $\mathrm{LF}(n=17$, $\mathrm{r}$-PLS $=0.669, P=0.22, Z=0.794$; Fig. 3b). Further, we found the effect sizes of jaw integration within TRC and LF to be statistically distinct ( $\mathrm{Z}$ pairwise $=1.678, P=0.047$ ). Altogether, our data support the assertion that the LOJ and LPJ are evolutionarily integrated at multiple taxonomic levels, but they also appear to indicate that certain taxa, such as Labeotropheus, 
can more readily generate adaptive morphological variation in each jaw complex independently.

Genetic basis for oral and pharyngeal jaw shape covariation. To understand whether phenotypic covariation between the LOJ and LPJ is genetically determined we performed a quantitative trait loci (QTL) analysis to identify prospective genomic regions involved in jaw shape variation for both the LOJ and LPJ. Specifically, we extended an existing genetic cross between the more strongly integrated TRC and the more weakly integrated LF to the $\mathrm{F}_{5}$ generation. Details of the pedigree may be found in ${ }^{34}$ and in the supplement. For this experiment, we genotyped $636 \mathrm{~F}_{5}$ hybrids and produced a genetic map containing 812 singlenucleotide polymorphisms (SNPs) spread across 24 linkage groups (Supplementary Data 6). With a total length of $1431 \mathrm{cM}$, our high-resolution linkage map contained a marker every $1.83 \mathrm{cM}$, on average, allowing us to leverage the increased number of recombination events that occurred to reach an $\mathrm{F}_{5}$ population. We then characterized LOJ and LPJ shape in $409 \mathrm{~F}_{5}$ hybrids using the same landmarking scheme described above, and performed a two-block PLS analysis. In concordance with our findings from natural populations, we documented an association between jaw complexes in this laboratory pedigree $(r-P L S=0.491, P=0.001$, effect size $=6.189$; Fig. 3c).

We next performed a PCA on the hybrid landmark configurations to distill the data down to a series of orthogonal axes that best explain shape variation among individuals. We extracted the first two PCs from the LOJ and LPJ as each axis represented more than $10 \%$ of the shape variation (Supplementary Data 7; Supplementary Figs. 4 and 5). The first axis of the LOJ reflected more general variation in depth, width, and length of the element ( $41.8 \%$ of variation), while the second axis reflected more specific variation in the length of the ascending arm of the articular--the process for which jaw closing muscles attach (12.7\% of variation). The first axis of the LPJ reflected width, length, and wing process size $(33.7 \%$ of variation), while the second axis reflected depth and the size of the anterior keel - the process for which the pharyngeal jaw pharyngohyoideus muscle attaches and controls jaw adduction (14.2\% of variation). We then utilized these PC scores as traits to run in our QTL analyses to investigate the genetic basis for variation in these structures.

QTL mapping implicates pleiotropic control of LOJ and LPJ shape variation. Integration between LOJ and LJP shapes in the $\mathrm{F}_{5}$ predicts that this pattern of covariation will be reflected in the genotype-phenotype map. Specifically, we predict that we will find overlapping QTL for both jaws. We used a multiple QTL mapping (MQM) approach to test this prediction. Specifically, we performed QTL scans for all four traits and quantitatively assessed the evidence for significant QTL marker(s) using a permutation procedure that reshuffles the phenotypic data relative to genotypic data 1000 times to generate a null distribution, disassociating any possible relationship between genotype and phenotype, to then compare the empirical distribution against ${ }^{35}$. Once candidate QTL markers were identified, we calculated an approximate Bayesian credible interval to determine the region in which a potential candidate locus would reside. We uncovered a total of five QTL for LOJ traits, and four QTL for LPJ traits (Fig. 4a; Supplementary Data 8). While most QTL localize to different linkage groups, we also identified some QTL that colocalized. Two traits (LOJ PC1, LPJ PC1) share a marker on LG4, while three traits (LOJ PC1, LOJ PC2, LPJ PC1) colocalized to the same markers on LG7. These data are consistent with pleiotropy on LG7 and possibly LG4.
We then quantitatively assessed the evidence for pleiotropy using a likelihood ratio test (LLRT) to compare the null hypothesis of a common pleiotropic QTL to the alternative hypothesis that they are affected by separate QTL ${ }^{36,37}$. The overlap on LG4 at a single marker $(43.57 \mathrm{cM})$ was deemed significant (LLRT $=1.85, P=0.03$ ), indicating that we can reject the null hypothesis and that these peaks likely represent separate QTL for each trait (Supplementary Fig. 6). The three traits that overlap on LG7 spanned two markers $(19.12 \mathrm{cM}-28.04 \mathrm{cM})$ and were all deemed non-significant (LOJpc1-LPJpc1: LLRT $=0.02$, $P=0.66$, Fig. 4b; LOJpc2-LPJpc1: LLRT $=0.20, P=0.41$, Fig. $4 \mathrm{c}$ ), leading us to accept the null hypothesis and conclude that this interval likely contains a single pleiotropic QTL. Whether a single gene, or multiple closely linked genes drive this pleiotropic signal requires a fine-mapping approach.

Notably, this locus on LG7 has been implicated previously in underlying LOJ and LPJ shape in another Lake Malawi cichlid cross between LF and Maylandia zebra ${ }^{38,39}$. Maylandia species, like Tropheops, were generally more integrated in our macroevolutionary analysis (Fig. 2a), and thus another cross between LF and a species with higher integration values point to the same locus. This suggests that the genetic mechanism of integration may be conserved.

Fine mapping identifies two candidate genes critical for bone formation. To gain insights into which gene(s) may be pleiotropically regulating LOJ and LPJ jaw shape variation on LG7 we constructed a fine map with greater marker density to investigate genotype-phenotype associations with greater resolution. To that end, we anchored QTL intervals to particular stretches of physical sequence of the Maylandia zebra genome ${ }^{40}$. We then identified additional RAD-seq SNPs across the linkage group of interest and genotyped them in the $F_{5}$. Based on this we created two fine maps: the first spanned the entirety of LG7 group with an average spacing of around one marker every $490 \mathrm{~kb}$ (Supplementary Data 9), the second matched the QTL marker range revealed by the Bayesian credible interval analysis with an average spacing of around one marker every $180 \mathrm{~kb}$ (Supplementary Data 10). We also calculated $\mathrm{F}_{\mathrm{ST}}$ from a panel of wild-caught LF $(n=20)$ and TRC $(n=20)$, and primarily focused on $\mathrm{F}_{\mathrm{ST}}$ values of 1.0 that would indicate complete segregation of a SNP between LF and TRC. At every marker on our LG7 fine maps, we calculated the difference in the values of our three colocalized traits between those hybrids homozygous for the LF allele and those homozygous for the TRC allele.

We identified a small region on LG7 that exhibited large differences in the average phenotypic effect of those hybrids with either LF or TRC alleles. In our full LG7 map we identified a $\sim 2 \mathrm{mb}$ region $(46.7 \mathrm{mb}-48.7 \mathrm{mb})$ that peaked in all three traits (Fig. 4d; Supplementary Data 11). Notably, the traces of all three traits across our LG7 fine maps track together in an almost identical fashion. In our fine map that centered on the Bayes credible interval, we found evidence for both large phenotypic effects among all traits, and the presence of several $\mathrm{F}_{\mathrm{ST}}$ markers approaching or equal to 1.0 (Fig. 4e; Supplementary Data 12). One marker combined an $\mathrm{F}_{\mathrm{ST}}$ score of 1.0, indicating complete segregation of that allele between LF and TRC, with high average phenotypic effects across all traits (Supplementary Fig. 7). This SNP fell within an intron of dymeclin $(d y m)$, a gene that is necessary for correct organization of Golgi apparatus and controls endochondral bone formation during early development. Dym is critical for chondrocyte development and previous research using the zebrafish model found an expression pattern that spanned the presumptive mandibular and ceratobranchial regions at larval stages ${ }^{41}$. Mutations in this gene lead to profound effects 
a

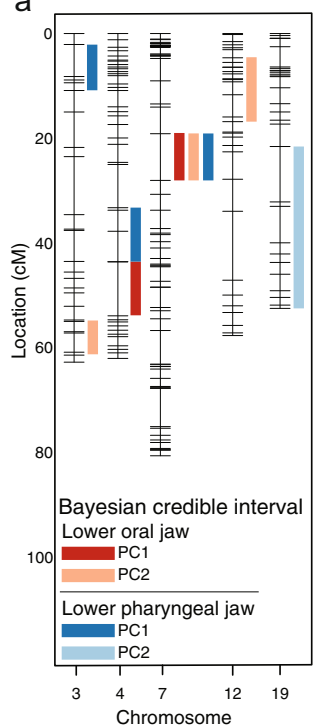

b

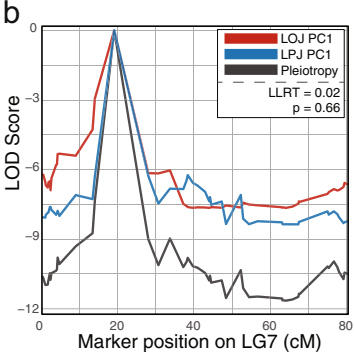

C

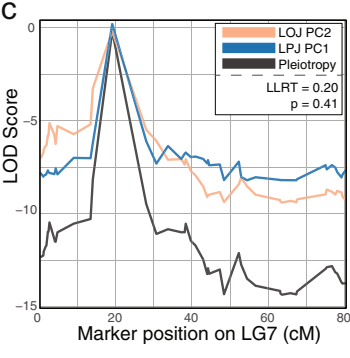

d
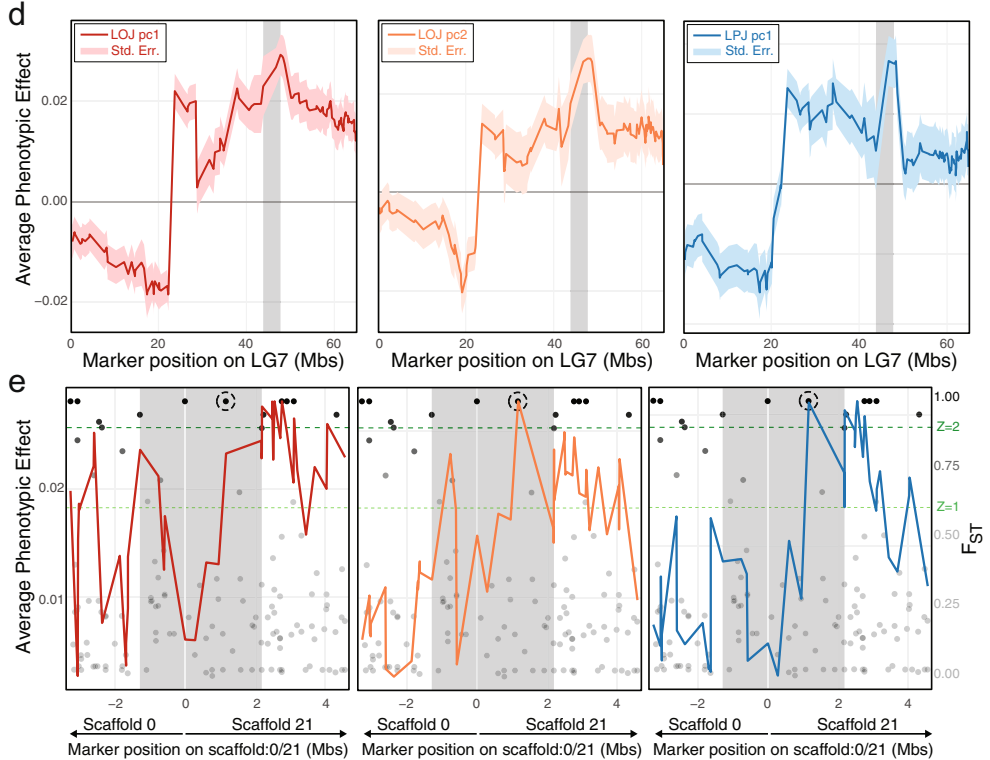

Fig. 4 Genetic analyses to identify regions of the genome responsible for major changes in jaw shape. All plots are based on 409 LFXTRC $F_{5}$ hybrids. a QTL analysis to identify positions in the genome most associated with each trait. b Pleiotropy analysis on linkage group seven to determine whether the oral jaw PC1 trait colocalizes to the same region as the pharyngeal jaw PC1 trait. Significance was determined using a likelihood ratio test (LLRT). c Pleiotropy analysis on linkage group seven to determine whether the oral jaw PC2 trait colocalizes to the same region as the pharyngeal jaw PC1 trait. Significance was determined using a LLRT. d Fine mapping all traits across the entirety of LG7. Values furthest from 0 reflect the largest differences between hybrids with LF and TRC genotypes at a given marker. We find peak genotype-phenotype association at $~ 50$ mb that coincides with our Bayes credible interval (grey bar). Intervals that surround the average phenotypic effect line denote standard error of the mean. e Fine mapping all traits across the Bayes credible interval. Population level genetic diversity $\left(F_{S T}\right)$ data are applied to the map (black dots) with the opacity of each SNP dependent on the degree of segregation between LF and TRC, with those falling above an empirical Z-score threshold of 0.6 determined to be significant, and those above 0.9 deemed highly significant (green lines). Within the credible interval there are four SNPs with $\mathrm{F}_{\mathrm{ST}}$ values of 1.0, but a single SNP that falls within a genotypephenotype peak residing within an intron of dym (black circle). Source data are provided as a Source Data file.

on the size and shape of bones due to misregulated chondrocyte development ${ }^{42}$. Just downstream ( $8 \mathrm{~kb}$, Supplementary Fig. 7) of dym is mothers against decapentaplegic homolog 7 (smad7), an antagonist of both TGF- $\beta$ and BMP signaling and a suppressor of bone formation. As an inhibitory Smad, smad7 negatively regulates genes from the BMP and TGF- $\beta$ signaling pathways (i.e., $b m p-2,-4,-7$, nodal, etc.) that are known to shape phenotypic differences in the craniofacial skeleton across a wide range of taxa including cichlids $25,38,43$, Geospiza finches ${ }^{44,45}$, and Anolis lizards ${ }^{46}$, primarily because these genes have the capacity to influence size in structures of trophic importance such as the mandible ${ }^{47}$. Both of these genes represent good candidates for controlling shape variation in the LOJ and the LPJ simultaneously. While two of the three traits peak at the dym SNP, when considering markers just outside the credible interval another peak is visible (especially for LOJ PC1) that sits close to notch $1 a$, a gene involved in skeletal development and homeostasis. Notch1a is flanked by two fully segregated $\mathrm{F}_{\mathrm{ST}}$ markers. The upstream marker is around $\sim 60 \mathrm{~kb}$ from the promoter region, while the downstream marker resides around $\sim 52 \mathrm{~kb}$ away from the gene within an intron of $k c n t 1$, a gene involved in potassium channel development that appears to regulate brain function ${ }^{48}$. While $k c n t 1$ reflects a poor candidate gene for our analysis, the intronic SNP could act as a distant enhancer of notch1a. Thus, given the combined results from QTL and fine-mapping, dym and smad7 represent strong candidates, but we cannot rule out notch1a.

Correlated expression of key genes between LOJ and LPJ. We used quantitative real-time PCR (qPCR) to assess and compare the expression levels of $d y m$, smad7, and notchla in the LOJ and LPJ of three mbuna genera from lake Malawi (Tropheops $n=6$, Labeotropheus $n=8$, Maylandia $n=8$ ). We used Labeotropheus and Tropheops to complement our quantitative genetic analysis, and all three taxa were represented in our phenotypic assessments of integration, permitting a comparison between macroevolutionary associations of the LOJ and LPJ with the underlying genetic architecture and expression for jaw complex correlation. We collected tissue samples from young juveniles of these four taxa, taking the LOJ and LPJ, alongside the caudal fin to act as an internal control, and performed a phenol/chloroform RNA extraction. We designed primers with high amplification efficiency (>90\%) for our three genes (Supplementary Data 13), and used $\beta$-actin as our control gene. We calculated relative expression of the LOJ and LPJ using the $2^{-\Delta \Delta C T} \operatorname{method}^{49}$, and compared expression across taxa and between tissues (Supplementary Data 14 and 15).

We initially compared tissue level expression levels between Labeotropheus and Tropheops and found small differences in dym expression, with LF typically exhibiting slightly higher levels $(t$ test LOJ $t=2.863, P=0.014$; LPJ $t=1.212, P=0.249$; Fig. 5a). These results are consistent with previous expression studies that demonstrated how Labeotropheus typically has up-regulated bone and collagen markers and as a consequence has greater bone deposition and a more robust craniofacial skeleton ${ }^{50,51}$. Expression level differences were also noted for notch1a and smad7 (Fig. 5b-c); both showed reduced expression in LF, which is expected based on each genes role as negative regulators of bone formation 52,53 . While the differences between species were fairly small in smad7 between taxa ( $t$-test LOJ $t=-1.869, P=0.086$; LPJ $t=-0.359, P=0.726)$, they were more notable in notchla $(t$ test LOJ $t=-1.947, P=0.080$; LPJ $t=-3.221, P=0.009$ ). Notchla is involved in skeletal remodeling, previous research has shown LF exhibits a minimal plastic response to environmental stimuli ${ }^{51}$. Thus, the relatively low expression of notch $1 a$ in 

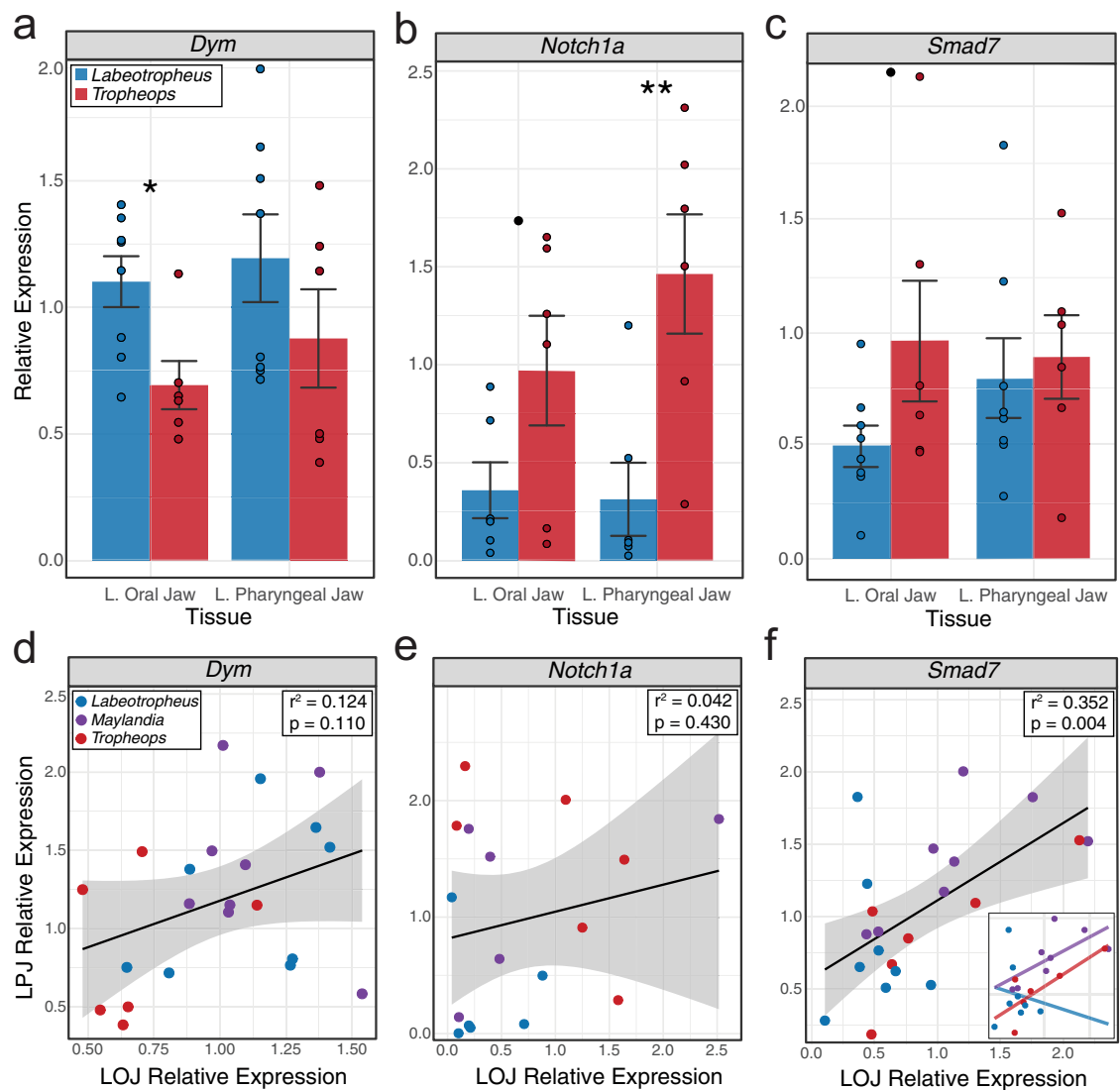

Fig. 5 Comparing expression levels of $\mathbf{d y m}$, smad7, and notch1a via qPCR in the oral and pharyngeal jaws. a dym bar plot; (b) notch1a bar plot; (c), smad7 bar plot; (d), dym scatter plot; (e), notch1a scatter plot; (f), smad7 scatter plot. a-c bar plots depict mean relative expression levels, error bars denote standard error. $\mathbf{d}$-f Scatterplots depict relative expression levels of the LOJ and LPJ, error bounds surrounding the linear regression line denote standard error. e inset, linear regression for each genus. Three cichlid taxa were included: Labeotropheus $n=8$, Tropheops $n=6$, Maylandia $n=8$. Bar plot significance determined via $t$-tests: $\bullet P<0.10,{ }^{\star} P<0.05,{ }^{\star} * P<0.01$. Source data are provided as a Source Data file.

Labeotropheus compared to Tropheops is consistent with this observation. While only representing a single life-history stage, the expression differences between species suggest that all three genes may underlie the development of species-specific shapes for the LOJ and/or LPJ. However, visualizing the data this way cannot speak to whether one or more of these loci underlie the covariation of the jaws.

To more explicitly address this question, we assessed the strength of correlation between LOJ and LPJ expression levels with all taxa. We found no evidence for correlated expression of $d y m$ between the LOJ and LPJ among taxa $\left(r^{2}=0.124, P=0.11\right.$; Fig. 5d), nor notch1a ( $r^{2}=0.042, P=0.43$; Fig. 5e), whereas we found a conspicuously strong correlation for $\operatorname{smad} 7\left(r^{2}=0.352\right.$, $P=0.0036$; Fig. 5f). These findings indicate that while $d y m$ and notch1a expression may be different between taxa and tissues, they do not appear to be strong candidates for driving coordinated change in the LOJ and LPJ. Alternatively, smad7 represents a more robust candidate for regulating the covariation between these two structures.

Another pattern that emerged from these data is that not all taxa appear to be contributing equally to correlations in gene expression. For smad7, Tropheops individuals fall close to the trend line, while Labeotropheus individuals do not. This observation is notable, because it agrees with our macro- and microevolutionary analyses, which demonstrated that Labeotropheus exhibits weak integration between the LOJ and LPJ compared to Tropheops (Figs. 2a, 3a, b). When quantitatively compared, we observed evidence of more correlated smad7 expression between the jaws in Tropheops $\left(r^{2}=0.641, P=0.056\right)$ and Maylandia $\left(r^{2}=0.452, P=0.068\right)$, but not in Labeotropheus $\left(r^{2}=0.02, P=0.74\right)$. Alternatively, $d y m$ expression in the LOJ and LPJ was not correlated in any taxa (Tropheops, $r^{2}=0.076$, $P=0.60 ; \quad$ Maylandia, $r^{2}=0.052, \quad P=0.59 ; \quad$ Labeotropheops, $\left.r^{2}=0.188, P=0.28\right)$. These data raise the interesting possibility that LF has evolved a mechanism to independently modulate expression of smad7 between the LOJ and LPJ, and that this could represent a means to facilitate the decoupling of jaw shape variation (Fig. 5f, inset). Operationally, this may involve varying expression in one jaw system, over the other. For example, Smad7 expression in the LOJ of Labeotropheus appears more constrained relative to Tropheops (Bartlett's $K^{2}=4.74, P=0.029$ ), while expression in the LPJ is similarly variable to Tropheops (Bartlett's $\left.K^{2}=0.048, P=0.826\right)$. Taken together, our expression data implicate $\operatorname{smad} 7$ as a putative pleiotropic regulator of LOJ and LPJ shape variation in cichlids, an observation that deserves future exploration. Further, dym and notch $1 a$ remain candidates for regulating variation in LOJ and LPJ shapes, especially given their broad roles in craniofacial bone development, regulation, and homeostasis.

\section{Discussion}

In spite of major advances in connecting genotype to phenotype, a considerable challenge in the field of evolutionary biology remains linking changes in the genome, to patterns of phenotypic evolution. One such open-ended question involves the origins of 
genetic, developmental, or functional constraints and their impact on evolutionary potential ${ }^{54}$. These constraints ultimately influence the availability of variation for natural selection to act upon, driving evolution in certain directions while limiting it in others (Fig. 1a). There are a growing number of empirical examples of constraints impacting evolvability, which have been described at a variety of levels, including developmental (e.g., the ubiquitous pentadactyl limb of tetrapods ${ }^{55}$ ), functional (e.g., force-velocity trade-offs in bird feeding ${ }^{56}$ ), and evolutionary (e.g., reduced disparity of marsupial forelimbs owing to a climbing neonate ${ }^{57}$ ). In an environment with ample ecological opportunity, breaking from these constraints can lead to a rapid increase in morphological diversity and the occupation of new, previously inaccessible, ecological niches. For example, the transition to powered flight in bats limited body size, and thus eye size, making the capture of insect prey more difficult; however, some bats circumvented this constraint by developing a specialized auditory and echolocation system, removing their sole reliance on vision for hunting ${ }^{58}$. Similarly, the release of genetic constraints within Labeotropheus cichlids permitted the development of a hypertrophied snout via an expansion of ligamentous and connective tissues to increase the efficiency of cropping filamentous algae from rocks ${ }^{43,59}$.

Another consequence of genetic, developmental, and functional constraints is phenotypic integration, whereby seemingly independent traits vary in unison. While determining the origins of integration has proved difficult (i.e., ${ }^{60}$ ), trait integration can facilitate or limit morphological evolution depending on the direction of selection ${ }^{2}$. While phenotypic integration is a population-level metric, integration can have far-reaching effects on macroevolutionary processes such as taxonomic diversification, extinction, and morphological evolution ${ }^{3,7}$. Indeed, there is an emerging consensus that phenotypic integration can facilitate rapid and coordinated trait evolution in teleosts ${ }^{5,61,62}$, and may reflect a general trend across organisms $s^{4,63,64}$. Within cichlids, a lack of functional integration between oral and pharyngeal jaws has enabled the separation of prey capture from prey processing, a mechanism that is considered key to their success ${ }^{15}$. However, we provide evidence that African cichlid trophic evolution has generally occurred within the context of a coupled jaw system, a trend that was also recently noted in New World cichlids ${ }^{14}$. We demonstrate further that LOJ-LPJ integration may be due, at least in part, to pleiotropy, a phenomenon that is commonly theorized to determine patterns of covariation and limit evolvability (i.e.., ${ }^{9,65}$ but $\operatorname{see}^{60}$ ). We postulate that integration between LOJ-LPJ shapes in cichlids is not a constraint, but rather an attribute that promotes rapid shifts in foraging niche ${ }^{33,66}$, an ability that should be particularly advantageous in young and/or dynamic environments similar to the East African Rift-Valley. If true, then LOJLPJ integration may be actively selected for, perhaps via pleiotropy. We also note the variation in the strength of LOJ-LPJ integration across rift lake cichlids, and that some taxa particularly foraging specialists (i.e., Labeotropheus, Trematocranus), exhibited weaker integration. This observation is consistent with the idea that pleiotropy itself can evolve, and the strength of integration that pleiotropy may create between traits is unlikely to be uniform across clades (reviewed $\mathrm{in}^{28}$ ). Thus, integration between the jaws of cichlids should not be considered a binary choice between "coupled" or "decoupled," but rather exist along a skewed distribution ${ }^{14}$, with most taxa exhibiting tight integration between the jaws, and a subset of foraging specialists exhibiting less (or differently) integrated jaws. These observations are consistent with the idea that foraging niche partitioning may underlie the evolution LOJ-LPJ integration. We predict that had we used a cross between two species that both exhibited strong jaw integration (i.e., Tropheops and Maylandia), more pleiotropic loci would have been uncovered. Understanding the mechanisms behind how Labeotropheus and other specialist cichlid taxa have evolutionarily decoupled their oral and pharyngeal jaws presents an interesting avenue for future exploration. More generally, documenting the genetic mechanisms behind jaw (de)coupling will be essential to understanding how the immensely diverse trophic morphology of cichlids originated and became a critical catalyst of the African cichlid radiations ${ }^{67}$.

\section{Methods}

Specimen Collection. Specimens used in our analyses were either imported directly from the wild, obtained through the aquarium trade and raised in the authors' fish facility, loaned from museums, or downloaded from online collection repositories. For our macroevolutionary analysis, we used 107 individual cichlids representing 88 taxa. We scanned 42 individuals representing 23 taxa, with trait values from most taxa based on measures taken from three individuals. We also downloaded 65 individuals representing 65 taxa from the online repository morphosource.org (Supplementary Data 1). For our microevolutionary study, we used 58 wild-caught Tropheops species representing 23 taxa, with 22 taxa present in the tree (Supplementary Data 4). When comparing jaw integration between LF and TRC, individuals were obtained from wild-caught and lab-raised populations. We scanned a total of 28 individuals: 17 LF and 11 TRC (Supplementary Data 5). Our hybrid mapping population between LF and TRC was generated in the lab and comprised 409 individuals (Supplementary Data 6 and 7). All experiments involving animals were performed in compliance with the Institutional Animal Care and Use Committee at UMass Amherst (\#2018-0094 to RCA).

uCT Scanning. We performed all $\mu \mathrm{CT}$ scanning using an X-Tek HMXST 225 (Nikon Corporation). All scans were acquired at 25-35 micron resolution using $80-125 \mathrm{kV}$ and $75-120 \mu \mathrm{A}$. We extracted $\mathrm{z}$-stack images and segmented the hard tissues using Mimics (v19 Materialise NV), before exporting the 3D models to Geomagic 2014 (v1.0 3D Systems). We then used Geomagic to digitally dissect the lower oral and pharyngeal jaws from the whole organism ready for $3 \mathrm{D}$ geometric morphometrics.

Geometric morphometric data collection. We used a series of fixed landmarks (LMs) to characterize shape information by placing LMs on both the left and right sides in both jaws, which allowed us to separate the symmetric from asymmetric components of shape variation (Supplementary Fig. 1a, b; Supplementary Data 16). We placed a suite of LMs at key functional and developmental positions (i.e., processes, muscle insertion points, sutures etc.) on the lower oral jaw and the lower pharyngeal jaw (Supplementary Fig. 1c, d). We used a total of 20 fixed LMs on the lower oral jaw and 15 fixed LMs on the lower pharyngeal jaw and landmarked both jaws on the left and right sides across the midlines to permit later corrections for asymmetry, a product of developmental noise. All jaws sampled in this study were digitized based on the same landmark configuration. A single individual (AJC) performed all landmark digitizing using Landmark Editor (v3.0) ${ }^{68}$ to eliminate inter-observer error. All subsequent morphometric and genetic analyses were performed using $R$ (v4.0.1) unless otherwise stated ${ }^{69}$.

For each of our four major analyses (macroevolutionary, microevolutionary, parentals, hybrids) we performed a Procrustes superimposition to remove the effects of translation, scaling, and rotation in our oral and pharyngeal jaw landmark

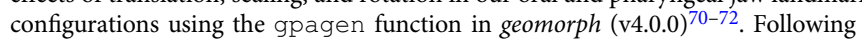
Procrustes superimposition, if multiple individuals were digitized from a single species in the macroevolutionary (across African cichlids) and microevolutionary (across Tropheops sp.) components of this study, we calculated a species mean landmark configuration. To assess the relationship between jaw size and shape in our landmark configurations we performed a Procrustes ANOVA between the centroid size and shape of each individual jaw using the geomorph procD. Im function or the procD.pgls function when comparing among species. We observed a small but significant degree of jaw allometry in many collections (Supplementary Data 17), and therefore extracted residual landmark coordinates from the Procrustes ANOVA to provide allometry-free jaw configurations for use in subsequent analyses.

To assess and correct for fluctuating asymmetry in our allometrically corrected lower oral and pharyngeal jaw landmark configurations we used the bilat.symmetry function in geomorph. All structures were found to exhibit significant degrees of directional asymmetry (Supplementary Data 18). We then extracted landmark configurations from both jaws based on the symmetric components of variation for use in each future analysis. Isolating the symmetric component of landmark variation should reduce the amount of developmental noise in our data and increase our ability to detect subtle genetic signals.

To examine the range of LOJ and LPJ morphologies among our African cichlids we performed a Principal Components Analysis (PCA) that reduced each jaw landmark dataset into a series of orthogonal axes that best describe variation among cichlid taxa (Supplementary Fig. 3; Supplementary Data 2 and 3). We performed PCA using the gm.prcomp function in geomorph. Given the large volume of morphospace occupied by those cichlids from Lakes Malawi and Tangyanika, we directly compared cichlid disparity between these lakes using 
Procrustes variance. We performed a phylogenetically corrected disparity analysis using the morphol.disparity function in geomorph.

Lower oral and pharyngeal jaw shape correlations. We examined the degree of association between our allometrically- and symmetrically corrected lower oral and pharyngeal jaw shapes using partial least squares (PLS) analysis. When assessing the degree of jaw shape covariation among taxa from across the rift lakes we corrected for the phylogenetic non-independence of our jaw traits by using a Bayesian time calibrated tree ${ }^{31}$, and incorporated that into our PLS using the phylo.integration function in geomorph to examine the degree of jaw association under a Brownian model of evolution ${ }^{71,73}$. Similarly, we used the same routines to conduct a phylogenetically corrected PLS on our collection of Lake Malawi Tropheops species from across deep and shallow habitats using an amplified fragment length polymorphism tree ${ }^{33}$. To assess the degree of jaw covariation within our two taxa of interest, TRC, and LF, and also within $\mathrm{F}_{5}$ hybrids of those species we again used PLS via the two.b.pls function in geomorph ${ }^{74,75}$. To compare integration between cichlids from Tanganyika and Malawi, and between LF and TRC, we used the compare.pls function in geomorph to statistically compare the effect sizes of our PLS analyses. While we list the correlation coefficient ( $r$-PLS) and the multivariate effect size in all cases, there is no direct correspondence between the dispersion of projected scores and variance explained by axes, as in a PCA, meaning there is not a direct analog of total variation explained to total covariation explained in the PLS ${ }^{76}$.

Quantitative Trait Loci. To begin constructing our genetic map we first crossed a wild-caught LF female from Makanjila Point with a TRC male from Chizumulu Island. We then interbred the resultant full-sibling $F_{1}$ family to produce an $F_{2}$ population for genotyping and the production of a genetic map ${ }^{34}$. Next, we performed further intercrosses by randomly interbreeding individuals from different families up to the $\mathrm{F}_{5}$ generation $(n=636)$. The additional intercrossing allowed this $\mathrm{F}_{5}$ hybrid population to undergo more recombination events, thus increasing the resolution of mapping intervals. We extracted and sequenced genomic DNA from caudal fin clips of $636 \mathrm{~F}_{5}$ hybrids following standard RAD-seq procedures ${ }^{34,77}$. As the $\mathrm{F}_{5}$ hybrid population did not exhibit a tractable pattern of Mendelian inheritance, we used a genetic map derived from the $\mathrm{F}_{2}$ generation of the same pedigree. In particular, we genotyped a subset of 812 evenly spaced genetic markers in the $F_{5}$ population, and used this map for all future QTL analyses. Full details of the cross and construction of the map can be found in the supplement.

To extract shape information from our $\mathrm{F}_{5}$ hybrid oral and pharyngeal jaws we performed a principal component (PC) analysis that serves to reduce our landmark coordinate data to a series of orthogonal axes that best describe shape variation among individuals. The first axis, or component, reflects the greatest degree of shape variation change in the jaws, and subsequent axes describe progressively less shape information. We used the gm.prcomp function in geomorph to perform our PC analysis ${ }^{71}$. We extracted the first two PCs from each jaw to use as traits in our QTL analysis to identify regions of the genome that may be responsible for controlling variation in that trait. Subsequent PC axes explained $<10 \%$ of the jaw shape variation. While PCs will typically capture large-scale changes in shape rather than, for example, the length of a particular process, we consider this a benefit as we are searching for loci that could bring about major correlated changes across jaw complexes.

To examine regions of the genome responsible for variation in lower oral and pharyngeal jaw shape we searched for putative loci using the multiple QTL mapping (MQM) approach ${ }^{78}$ from $r / q t l(\mathrm{vl} .46-2)^{79}$. We performed a QTL scan via the scanone function to examine initial QTL peaks indicative of genotypephenotype associations. We then manually added cofactors to the model based on the location of QTL peaks before performing a MQM analysis using the momscan function. As more cofactors were added, the momscan function determined cofactor fit via maximum-likelihood backward elimination. Cofactors were continually added to the model until we maximized the logarithm of odds (LOD) score. We then quantitatively assessed the significance of a QTL peak using the mqmpermutation function. The mqmpermutation function generates a null distribution of LOD scores at each marker by reshuffling the phenotypic data relative to genotypic data 1000 times $^{35}$. QTL marker LOD scores that exceed a 5\% LOD threshold are deemed significant. We then used the bayesint function to calculate an approximate Bayesian credible interval around a significant QTL marker for which a potential candidate gene responsible for shape variation would reside within

Bayesian credible intervals that overlap among traits may be indicative of pleiotropic control. To quantitatively determine whether a pair of traits with overlapping credible intervals are pleiotropic or are associated with separate QTL, we used a likelihood ratio test ${ }^{37}$. This test compares the QTL position that maximizes the likelihood under pleiotropy at a common QTL location, to a pair of QTL positions that maximize likelihood under a model where QTL locations are distinct. The logarithm of the ratio between these two likelihood values is the test statistic. In this test, traits localizing to a common QTL reflect the null hypothesis, while traits localizing to distinct QTL reflect the alternative hypothesis. Significance testing of pleiotropy vs. separate QTL was obtained via parametric bootstrapping ${ }^{80}$. We performed the pleiotropy and bootstrapping tests using the scan_pvl function in the qtl2pleio (v1.4.3) R package ${ }^{36,81}$.
Fine Mapping. Once we identified a candidate pleiotropic marker on linkage group seven we then identified additional RAD-seq SNPs in the $\mathrm{F}_{5}$ population and used them to map either the entirety of a linkage group (LG) or to a region defined by the Bayesian credible interval on a LG. We used the Maylandia zebra (MZ) genome to anchor QTL intervals to particular stretches of physical sequence along LG7 $7^{40}$ We utilized two different MZ genomes in our fine-mapping as the most recent version (MZ UMD2a) enabled high-density sampling across LG7 (1 marker every $490 \mathrm{~kb}$ ), while an older version (MZ UMD1.1) allowed us to combine mapping the credible interval with calculations of genetic divergence (1 marker every $180 \mathrm{~kb}$ ). When mapping the credible interval the QTL marker range spanned two contiguous stretches of the MZ genome located on subsections of Scaffold 0 and Scaffold 21. We mapped larger regions of Scaffold 0 and 21 that contained the interval and extended beyond it on either side by $\sim 200 \mathrm{~kb}$. We used the VCFtools package (v0.1.16) to extract markers from a given linkage group or scaffold and recode into smaller VCF files ${ }^{82}$, and then we used the $v c f R$ (v1.10.0) package in R to translate these VCF files into genotypic information that is readable by $r / q t^{83}$. Once our fine maps were complete we merged in our trait data and examined the difference in average trait values between $\mathrm{F}_{5}$ hybrids with the LF allele and TRC allele at every marker position using the effectsplot function in r/qtl.

Finally, we assessed the degree of genetic divergence between wild-caught LF $(n=20)$ and TRC $(n=20)$ populations to identify regions in the genome that are nearing the alternate fixation of distinct alleles ${ }^{34}$. This entailed calculating $\mathrm{F}$ statistic estimates $\left(\mathrm{F}_{\mathrm{ST}}\right)$ at each loci ${ }^{84}$. A large $\mathrm{F}_{\mathrm{ST}}$ value $(\sim 1.0)$ indicates high differentiation among populations at a given locus. We calculated $Z$-transformed $\mathrm{F}_{\mathrm{ST}}$ values $\left(\mathrm{zF}_{\mathrm{ST}}\right)$ from markers across the genome to obtain thresholds for cichlid divergence. We plotted two thresholds on our fine maps $(Z=2 ; Z=1)$ to illustrate markers undergoing divergence (Fig. 4e). In our cichlid populations, a $Z$-score of 1 equated to an $\mathrm{F}_{\mathrm{ST}}$ score of $\sim 0.6$, a value similar to that observed across other cichlid populations ${ }^{85}$.

Quantitative real-time PCR. We performed qPCR to assess expression level differences in three genes of interest and to examine the correlation in expression levels between lower oral and pharyngeal jaws. We focused on three well-studied genera in the lab, Labeotropheus, Tropheops, and Maylandia, that exhibit a continuum of oral and pharyngeal jaw shapes. Expression level analyses were performed on Labeotropheus fuelleborni (LF, $n=8$ ), Maylandia callainos (MC, $n=8$ ), and Tropheops kumara (TK, $n=6$ ) obtained from the aquarium trade. All animals were juveniles when sacrificed (LF standard length (SL), $40.4 \mathrm{~mm}-48.2 \mathrm{~mm}$; MC SL, $44.9 \mathrm{~mm}-54.6 \mathrm{~mm}$; TK SL, $44.3 \mathrm{~mm}, 61.2 \mathrm{~mm}$ (Supplementary Data 19). See the supplement for more information on fish facility conditions and animal husbandry protocols.

We dissected out the lower oral and pharyngeal jaws of our cichlids and took a clip of the caudal fin to perform phenol/chloroform-based RNA extractions. We performed a reverse transcriptase PCR on our extracted RNA to produce complementary DNA (cDNA) for qPCR. We designed primer pairs for $d y m$, notchla, and smad7, and used a previously published primer sequence for $\beta$-actin as our control gene $e^{43}$. We then checked primer efficiency using the standard curve method. We performed a GPCR on all our samples and calculated relative jaw expression following the $2^{-\Delta \Delta \mathrm{CT}}$ method ${ }^{49}$ using the pcr (v1.2.2) package in $\mathrm{R}^{86}$ We used $t$-tests to determine significance in expression level differences between LF and TRC, and then performed linear regression to examine the degree of correlation in expression levels. See the supplement for full details of the molecular analysis.

Reporting summary. Further information on research design is available in the Nature Research Reporting Summary linked to this article.

\section{Data availability}

Additional data can be found in the supplementary information. RADseq data can be downloaded from Figshare, https://doi.org/10.6084/m9.figshare.15831834.v1. Source data are provided with this paper.

\section{Code availability}

Scripts and raw data can be found on GitHub ${ }^{87}$, https://github.com/andrewjohnconith/ cichlid_OJPJ.

Received: 21 February 2021; Accepted: 30 August 2021;

Published online: 16 September 2021

\section{References}

1. Felice, R. N., Randau, M. \& Goswami, A. A fly in a tube: macroevolutionary expectations for integrated phenotypes. Evolution 72, 2580-2594 (2018).

2. Klingenberg, C. P. Evolution and development of shape: integrating quantitative approaches. Nat. Rev. Genet. 11, 623-635 (2010). 
3. Goswami, A., Smaers, J. B., Soligo, C. \& Polly, P. D. The macroevolutionary consequences of phenotypic integration: from development to deep time. Philos. Trans. R. Soc. B Biol. Sci. 369, 20130254 (2014).

4. Conith, A. J., Meagher, M. A. \& Dumont, E. R. The influence of climatic variability on morphological integration, evolutionary rates, and disparity in the carnivora. Am. Nat. 191, 704-715 (2018)

5. $\mathrm{Hu}, \mathrm{Y}$. et al. Evolution in an extreme environment: developmental biases and phenotypic integration in the adaptive radiation of antarctic notothenioids. BMC Evol. Biol. 16, 142 (2016).

6. Wagner, G. P., Pavlicev, M. \& Cheverud, J. M. The road to modularity. Nat. Rev. Genet. 8, 921-931 (2007).

7. Goswami, A., Binder, W. J., Meachen, J. \& O'Keefe, F. R. The fossil record of phenotypic integration and modularity: a deep-time perspective on developmental and evolutionary dynamics. Proc. Natl Acad. Sci. USA 112, 4891-4896 (2015).

8. Parsons, K. J., Márquez, E. \& Albertson, R. C. Constraint and opportunity: the genetic basis and evolution of modularity in the cichlid mandible. Am. Nat. 179, 64-78 (2012).

9. Hallgrímsson, B. et al. Deciphering the palimpsest: studying the relationship between morphological integration and phenotypic covariation. Evolut. Biol. 36, 355-376 (2009).

10. Hallgrimsson, B. et al. The developmental-genetics of canalization. Semin. Cell Dev. Biol. 88, 67-79 (2019).

11. Schluter, D. Adaptive radiation along genetic lines of least resistance. Evolution 50, 1766-1774 (1996).

12. Melo, D., Porto, A., Cheverud, J. M. \& Marroig, G. Modularity: genes, development, and evolution. Annu. Rev. Ecol. Evol. Syst. 47, 463-486 (2016).

13. Wainwright, P. C. Functional versus morphological diversity in macroevolution. Annu. Rev. Ecol. Evol. Syst. 38, 381-401 (2007).

14. Burress, E. D., Martinez, C. M. \& Wainwright, P. C. Decoupled jaws promote trophic diversity in cichlid fishes. Evolution 74, 950-961 (2020).

15. Wainwright, P. C. et al. The evolution of pharyngognathy: a phylogenetic and functional appraisal of the pharyngeal jaw key innovation in labroid fishes and beyond. Syst. Biol. 61, 1001-1027 (2012).

16. Matschiner, M., Böhne, A., Ronco, F. \& Salzburger, W. The genomic timeline of cichlid fish diversification across continents. Nat. Commun. 11, 5895 (2020).

17. Meier, J. I. et al. Ancient hybridization fuels rapid cichlid fish adaptive radiations. Nat. Commun. 8, 14363 (2017).

18. Meier, J. I. et al. The coincidence of ecological opportunity with hybridization explains rapid adaptive radiation in Lake Mweru cichlid fishes. Nat. Commun. 10, 5391 (2019).

19. Darrin Hulsey, C. Function of a key morphological innovation: fusion of the cichlid pharyngeal jaw. Proc. R. Soc. B Biol. Sci. 273, 669-675 (2006).

20. Liem, K. F. \& Osse, J. W. M. Biological versatility, evolution, and food resource exploitation in African Cichlid Fishes. Am. Zool. 15, 427-454 (1975).

21. Liem, K. F. Evolutionary strategies and morphological innovations: cichlid pharyngeal jaws. Syst. Zool. 22, 425-441 (1973).

22. Hulsey, C. D., García de León, F. J. \& Rodiles-Hernández, R. Micro- and macroevolutionary decoupling of cichlid jaws: a test of Liem's key innovation hypothesis. Evolution 60, 2096-2109 (2006).

23. Farina, S. C., Kane, E. A. \& Hernandez, L. P. Multifunctional structures and multistructural functions: integration in the evolution of biomechanical systems. Integr. Comp. Biol. 59, 338-345 (2019).

24. Burress, E. D. \& Muñoz, M. M. Ecological limits on the decoupling of prey capture and processing in fishes. Integr. Comp. Biol. icab148 https://doi.org/ 10.1093/icb/icab148 (2021).

25. Fraser, G. J. et al. An ancient gene network is co-opted for teeth on old and new jaws. PLOS Biol. 7, el000031 (2009).

26. Ahi, E. P., Singh, P., Duenser, A., Gessl, W. \& Sturmbauer, C. Divergence in larval jaw gene expression reflects differential trophic adaptation in haplochromine cichlids prior to foraging. BMC Evol. Biol. 19, 150 (2019).

27. Hulsey, C. D. et al. The integrated genomic architecture and evolution of dental divergence in East African cichlid fishes (Haplochromis chilotes x H nyererei). G3 Genes|Genomes|Genet. 7, 3195-3202 (2017).

28. Pavličev, M. \& Cheverud, J. M. Constraints evolve: context dependency of gene effects allows evolution of pleiotropy. Annu. Rev. Ecol. Evol. Syst. 46, 413-434 (2015).

29. Irschick, D. J. et al. Evo-devo beyond morphology: from genes to resource use. Trends Ecol. Evol. 28, 267-273 (2013).

30. Parsons, K. J. \& Albertson, R. C. Unifying and generalizing the two strands of evo-devo. Trends Ecol. Evol. 28, 584-591 (2013).

31. McGee, M. D. et al. The ecological and genomic basis of explosive adaptive radiation. Nature 586, 75-79 (2020).

32. Albertson, R. C. Morphological divergence predicts habitat partitioning in a lake malawi cichlid species complex. Copeia 2008, 689-698 (2008).

33. Conith, A. J., Kidd, M. R., Kocher, T. D. \& Albertson, R. C. Ecomorphological divergence and habitat lability in the context of robust patterns of modularity in the cichlid feeding apparatus. BMC Evol. Biol. 20, 95 (2020).
34. Albertson, R. C. et al. Genetic basis of continuous variation in the levels and modular inheritance of pigmentation in cichlid fishes. Mol. Ecol. 23, 5135-5150 (2014)

35. Arends, D., Prins, P., Jansen, R. C. \& Broman, K. W. R/qtl: high-throughput multiple QTL mapping. Bioinformatics 26, 2990-2992 (2010).

36. Boehm, F. J., Chesler, E. J., Yandell, B. S. \& Broman, K. W. Testing pleiotropy vs. separate QTL in multiparental populations. G3 Genes|Genomes|Genet. 9, 2317-2324 (2019).

37. Jiang, C. \& Zeng, Z. B. Multiple trait analysis of genetic mapping for quantitative trait loci. Genetics 140, 1111-1127 (1995).

38. Albertson, R. C., Streelman, J. T., Kocher, T. D. \& Yelick, P. C. Integration and evolution of the cichlid mandible: the molecular basis of alternate feeding strategies. Proc. Natl Acad. Sci. USA 102, 16287-16292 (2005).

39. Zogbaum. L. \& Albertson, R. C. Genetic Basis of Cichlid Pharyngeal Jaw Divergence: A Microhabitat Perspective. Integr. Comp. Biol. 57, e186 (2017).

40. Conte, M. A. \& Kocher, T. D. An improved genome reference for the African cichlid, Metriaclima zebra. BMC Genomics 16, 724 (2015).

41. Denais, C. et al. Dymeclin, the gene underlying Dyggve-Melchior-Clausen syndrome, encodes a protein integral to extracellular matrix and golgi organization and is associated with protein secretion pathways critical in bone development. Hum. Mutat. 32, 231-239 (2011).

42. Osipovich, A. B., Jennings, J. L., Lin, Q., Link, A. J. \& Ruley, H. E Dyggve-Melchior-Clausen syndrome: Chondrodysplasia resulting from defects in intracellular vesicle traffic. Proc. Natl Acad. Sci. USA 105, 16171-16176 (2008).

43. Conith, M. R. et al. Genetic and developmental origins of a unique foraging adaptation in a Lake Malawi cichlid genus. Proc. Natl Acad. Sci. USA 115, 7063-7068 (2018).

44. Abzhanov, A., Protas, M., Grant, B. R., Grant, P. R. \& Tabin, C. J. Bmp4 and Morphological Variation of Beaks in Darwin's Finches. Science 305, 1462-1465 (2004)

45. Mallarino, R. et al. Two developmental modules establish 3D beak-shape variation in Darwin's finches. Proc. Natl Acad. Sci. USA 108, 4057-4062 (2011).

46. Sanger, T. J. et al. The oestrogen pathway underlies the evolution of exaggerated male cranial shapes in Anolis lizards. Proc. Biol. Sci. 281, 20140329 (2014).

47. Parsons, K. J. \& Albertson, R. C. Roles for Bmp4 and CaM1 in shaping the jaw: evo-devo and beyond. Annu. Rev. Genet. 43, 369-388 (2009).

48. Quraishi, I. H. et al. Impaired motor skill learning and altered seizure susceptibility in mice with loss or gain of function of the Kcntl gene encoding Slack (KNa1.1) Na+-activated K+ channels. Sci. Rep. 10, 3213 (2020).

49. Livak, K. J. \& Schmittgen, T. D. Analysis of relative gene expression data using real-time quantitative PCR and the $2-\Delta \Delta \mathrm{CT}$ method. Methods 25, 402-408 (2001).

50. Roberts, R. B., Hu, Y., Albertson, R. C. \& Kocher, T. D. Craniofacial divergence and ongoing adaptation via the hedgehog pathway. Proc. Natl Acad. Sci. USA 108, 13194-13199 (2011).

51. Parsons, K. J., Taylor, A. T., Powder, K. E. \& Albertson, R. C. Wnt signalling underlies the evolution of new phenotypes and craniofacial variability in Lake Malawi cichlids. Nat. Commun. 5, 1-11 (2014).

52. Zanotti, S. \& Canalis, E. Notch Signaling and the Skeleton. Endocr. Rev. 37, 223-253 (2016)

53. Yan, X., Liu, Z. \& Chen, Y. Regulation of TGF- $\beta$ signaling by Smad7. Acta Biochim. Biophys. Sin. (Shanghai). 41, 263-272 (2009).

54. Young, N. M. et al. Embryonic bauplans and the developmental origins of facial diversity and constraint. Development 141, 1059 LP-1051063 (2014)

55. Stewart, T. A. et al. Evidence against tetrapod-wide digit identities and for a limited frame shift in bird wings. Nat. Commun. 10, 3244 (2019).

56. Herrel, A., Podos, J., Vanhooydonck, B. \& Hendry, A. P. Force-velocity tradeoff in Darwin's finch jaw function: a biomechanical basis for ecological speciation? Funct. Ecol. 23, 119-125 (2009).

57. Sears, K. E. Constraints on the morphological evolution of marsupial shoulder girdles. Evolution 58, 2353-2370 (2004)

58. Thiagavel, J. et al. Auditory opportunity and visual constraint enabled the evolution of echolocation in bats. Nat. Commun. 9, 98 (2018).

59. Conith, M. R., Conith, A. J. \& Albertson, R. C. Evolution of a soft-tissue foraging adaptation in African cichlids: Roles for novelty, convergence, and constraint. Evolution 73, 2072-2084 (2019).

60. Conith, A. J., Hope, S. A., Chhouk, B. H. \& Craig Albertson, R. Weak genetic signal for phenotypic integration implicates developmental processes as major regulators of trait covariation. Mol. Ecol. 30, 464-480 (2021).

61. Evans, K. M. et al. Integration drives rapid phenotypic evolution in flatfishes Proc. Natl Acad. Sci. USA 118, e2101330118 (2021).

62. Smith, A. J., Nelson-Maney, N., Parsons, K. J., James Cooper, W. \& Craig Albertson, R. Body shape evolution in sunfishes: divergent paths to accelerated rates of speciation in the centrarchidae. Evol. Biol. 42, 283-295 (2015). 
63. Watanabe, A., Balanoff, A. M., Gignac, P. M., Gold, M. E. L. \& Norell, M. A. Novel neuroanatomical integration and scaling define avian brain shape evolution and development. Elife 10, e68809 (2021).

64. Watanabe, A. et al. Ecomorphological diversification in squamates from conserved pattern of cranial integration. Proc. Natl Acad. Sci. USA 116 14688-14697 (2019).

65. Cheverud, J. M. Developmental integration and the evolution of pleiotropy. Am. Zool. 36, 44-50 (1996).

66. Hedrick, B. P. et al. Morphological diversification under high integration in a hyper diverse mammal clade. J. Mamm. Evol. 27, 563-575 (2020).

67. Salzburger, W. Understanding explosive diversification through cichlid fish genomics. Nat. Rev. Genet. 19, 705-717 (2018).

68. Wiley, D. F. et al. Evolutionary Morphing. In Proceedings of IEEE Visualization 431-438 (2005)

69. R Core Team. R: A Language and Environment for Statistical Computing. (2020).

70. Rohlf, F. J. On applications of geometric morphometrics to studies of ontogeny and phylogeny. Syst. Biol. 47, 147-167 (1998).

71. Adams, D. C., M. L. Collyer, A. Kaliontzopoulou, and E.K. Balken. 2021. Geomorph: Software for geometric morphometric analyses. R package version 4.0. (2021).

72. Adams, D. C. \& Otárola-Castillo, E. Geomorph: an R package for the collection and analysis of geometric morphometric shape data. Methods Ecol. Evol. 4, 393-399 (2013).

73. Adams, D. C. \& Felice, R. N. Assessing trait covariation and morphological integration on phylogenies using evolutionary covariance matrices. PLoS One 9, e94335 (2014).

74. Rohlf, F. J. \& Corti, M. Use of two-block partial least-squares to study covariation in shape. Syst. Biol. 49, 740-753 (2000).

75. Adams, D. C. \& Collyer, M. L. On the comparison of the strength of morphological integration across morphometric datasets. Evolution 70, 2623-2631 (2016).

76. Collyer, M. L. \& Adams, D. C. Phylogenetically aligned component analysis. Methods Ecol. Evol. 12, 359-372 (2021).

77. Chutimanitsakun, Y. et al. Construction and application for QTL analysis of a Restriction Site Associated DNA (RAD) linkage map in barley. BMC Genomics 12, 4 (2011).

78. Broman, K. W. \& Sen, S. A guide to QTL mapping with R/qtl. (SpringerVerlag, 2009).

79. Broman, K. W. \& Wu, H. QTL: Tools for Analyzing QTL Experiments. (2020).

80. Tian, J. et al. The Dissection of Expression Quantitative Trait Locus Hotspots. Genetics 202, 1563 LP-1561574 (2016).

81. Broman, K. W. et al. R/qt12: software for mapping quantitative trait loci with highdimensional data and multiparent populations. Genetics 211, 495-502 (2019).

82. Danecek, P. et al. The variant call format and VCFtools. Bioinformatics 27, 2156-2158 (2011).

83. Knaus, B. J. \& Grünwald, N. J. vcfr: a package to manipulate and visualize variant call format data in R. Mol. Ecol. Resour. 17, 44-53 (2017).

84. Nei, M. Molecular Evolutionary Genetics. (Columbia University Press, 1987).

85. Mims, M. C., Darrin Hulsey, C., Fitzpatrick, B. M. \& Todd Streelman, J. Geography disentangles introgression from ancestral polymorphism in Lake Malawi cichlids. Mol. Ecol. 19, 940-951 (2010).

86. Ahmed, M. \& Kim, D. R. pcr: an R package for quality assessment, analysis and testing of qPCR data. PeerJ 6, e4473 (2018).
87. Conith, A. J. \& Albertson, R. C. The cichlid oral and pharyngeal jaws are evolutionarily and genetically coupled. github.com/andrewjohnconith/ cichlid_OJPJ https://doi.org/10.5281/zenodo.5421511 (2021).

88. Malinsky, M. et al. Whole-genome sequences of Malawi cichlids reveal multiple radiations interconnected by gene flow. Nat. Ecol. Evol. 2, 1940-1955 (2018).

\section{Acknowledgements}

We thank Greg Lin at Harvard for $\mu \mathrm{CT}$ access, Daniel Pulaski for assistance with segmentation software, and Prakrit Subba for contributing initial pilot data to inform the experimental design. This work was funded by the National Institutes of Health \#R01DE026446 to RCA.

\section{Author contributions}

A.J.C. and R.C.A. formulated research plan and experimental design. A.J.C. performed all statistical and genetic analyses. A.J.C. and R.C.A. wrote the manuscript.

\section{Competing interests}

The authors declare no competing interests.

\section{Additional information}

Supplementary information The online version contains supplementary material available at https://doi.org/10.1038/s41467-021-25755-5.

Correspondence and requests for materials should be addressed to Andrew J. Conith or R. Craig Albertson.

Peer review information Nature Communications thanks Peter Wainwright and the other, anonymous, reviewers for their contribution to the peer review of this work. Peer reviewer reports are available.

Reprints and permission information is available at http://www.nature.com/reprints

Publisher's note Springer Nature remains neutral with regard to jurisdictional claims in published maps and institutional affiliations.

Open Access This article is licensed under a Creative Commons Attribution 4.0 International License, which permits use, sharing, adaptation, distribution and reproduction in any medium or format, as long as you give appropriate credit to the original author(s) and the source, provide a link to the Creative Commons license, and indicate if changes were made. The images or other third party material in this article are included in the article's Creative Commons license, unless indicated otherwise in a credit line to the material. If material is not included in the article's Creative Commons license and your intended use is not permitted by statutory regulation or exceeds the permitted use, you will need to obtain permission directly from the copyright holder. To view a copy of this license, visit http://creativecommons.org/ licenses/by/4.0/.

(C) The Author(s) 2021 